OPEN ACCESS

Edited by:

Pasquale Parisi,

Sapienza University of Rome, Italy

Reviewed by:

Vincenzo Santinelli,

IRCCS Policlinico San Donato, Italy Aleksandra Jezela-Stanek National Institute of Tuberculosis and

Lung Diseases, Poland

*Correspondence:

Xue-Mei Wu

xmwu@jlu.edu.cn

Specialty section:

This article was submitted to

Pediatric Neurology,

a section of the journal

Frontiers in Pediatrics

Received: 27 April 2021

Accepted: 02 June 2021

Published: 01 July 2021

Citation:

Liang J-M, Xin C-J, Wang G-L and Wu X-M (2021) Case Report: m.13513 G>A Mutation in a Chinese

Patient With Both Leigh Syndrome and Wolff-Parkinson-White Syndrome.

Front. Pediatr. 9:700898.

doi: 10.3389/fped.2021.700898

\section{Case Report: m.13513 G>A Mutation in a Chinese Patient With Both Leigh Syndrome and Wolff-Parkinson-White Syndrome}

\author{
Jian-Min Liang ${ }^{1,2}$, Cui-Juan Xin ${ }^{1}$, Guang-Liang Wang ${ }^{3}$ and Xue-Mei Wu ${ }^{1,2 *}$ \\ ${ }^{1}$ Department of Pediatric Neurology of Jilin University, Changchun, China, ${ }^{2}$ Jilin Provincial Key Laboratory of Pediatric \\ Neurology, Changchun, China, ${ }^{3}$ Department of Cardiology, Dalinghe Hospital of Far Eastern Horizon, Linghai, China
}

A number of causative mutations in mitochondrial and nuclear DNA have been identified for Leigh syndrome, a neurodegenerative encephalopathy, including m. $8993 \mathrm{~T}>\mathrm{G}$, m.8993 $\mathrm{T}>\mathrm{C}$, and m.3243A>G mutations in the MTATP6, MTATP6, and MT-TL1 genes, respectively, which have been reported in Leigh syndrome patients in China. The m.13513 G>A mutation has been described only a few times in the literature and not previously reported in China. Here we report the case of a 15-month-old boy who presented with ptosis and developmental delay and was diagnosed with Leigh syndrome and well as Wolff-Parkinson-White (WPW) syndrome. The m.13513 G>A mutation was found in DNA from blood. He was intubated due to respiratory failure and died at 23 months of age. The m.13513 G>A mutation in the ND5 gene of mitochondrial DNA is associated with Leigh syndrome and WPW syndrome; however, this is the first report of this mutation in a patient in China, highlighting the geographical and racial variability of Leigh syndrome.

Keywords: Leigh syndrome, 13513 mutation, Wolff-Parkinson-White syndrome 2, neurology, pediatric

\section{INTRODUCTION}

Leigh syndrome is a neurodegenerative disorder characterized by bilateral symmetrical necrotic lesions in the basal ganglia and brainstem that is typically identified within the first year of life (1). In China, a common causative mutation for Leigh syndrome is the SURF1 mutation, and m.8993 T>G, m.8993 T>C, and m.3243A>G mutations in the MTATP6, MTATP6, and MT-TL1 genes also have been identified as causative mutations in Chinese patients with Leigh syndrome (2). Another mutation, the m.13513 G>A mutation, is more commonly associated with MELAS (Mitochondrial Encephalopathy, Lactic Acidosis, and Stroke-like episodes) (3, 4), but $\sim 7 \%$ of Leigh syndrome cases in Western countries are estimated to be caused by the m.13513 G>A mutation (5). However, this mutation has not previously been reported in China in association with Leigh syndrome. Here we present the case of a 15-month-old boy in whom the G-to-A transition at nucleotide 13513 of the mitochondrial ND5 gene was identified and who died at the age of 23 months. As the first report of this mutation in China, it highlights the geographical and racial variability of Leigh syndrome. 

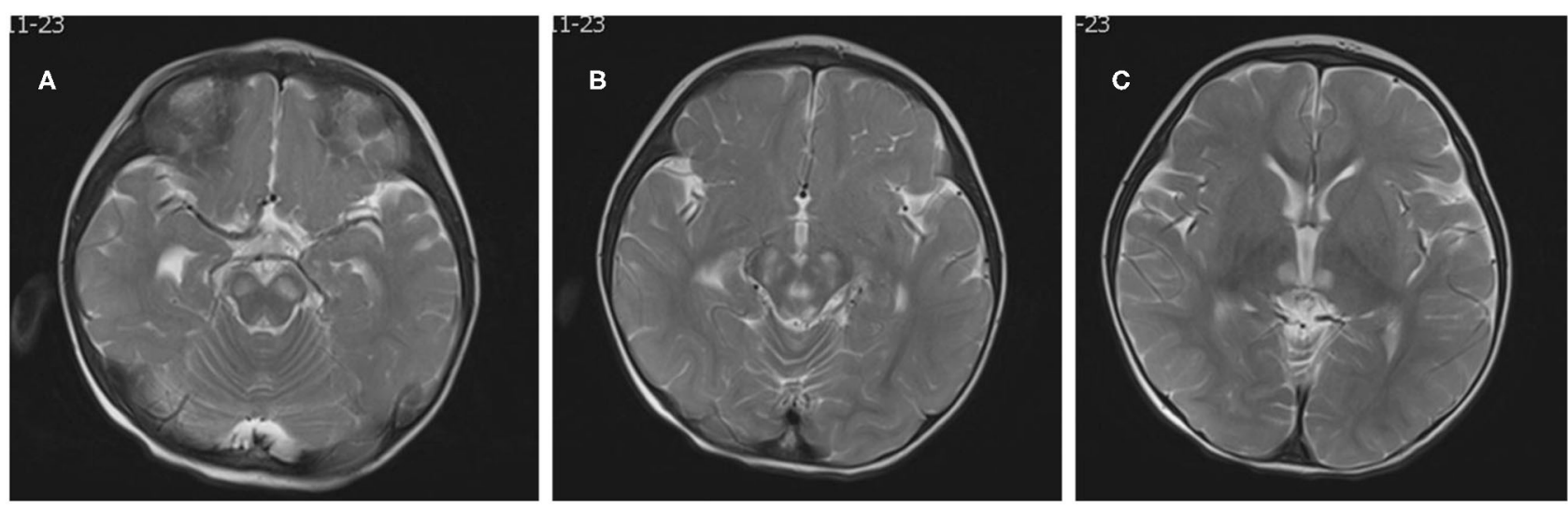

FIGURE 1 | Neuroradiological features. Brain MRI showed bilateral, symmetric signal abnormalities in the basal ganglia, and brain stem (A-C: T2-weighted images).

\section{CASE DESCRIPTION}

The patient was a 15-month-old boy. He was delivered by cesarean section because of a low level of amniotic fluid at 39 weeks of gestational age, with a birth weight of $2,540 \mathrm{~g}$. To the time of presentation, he had experienced poor body weight gain and delayed gross motor development. He could not sit up without support. His mother was clinically normal. At the age of 15 months, he was brought to our outpatient clinic for ptosis and developmental regression. On admission, his body weight was $8.1 \mathrm{~kg}$ (normal weight range $9.8-12.2 \mathrm{~kg}$ ) and body length was $75.5 \mathrm{~cm}$ (normal body length range 78.2$84 \mathrm{~cm}$ ). He could say simple words such as "papa" and "mama." Bilateral ptosis was found, but no obvious optic atrophy was detected by an ophthalmologist. Bilateral knee jerk was also exaggerated. Initial laboratory studies revealed normal complete blood counts as well as liver aspartase, blood urea nitrogen, creatinine, and electrolyte levels within normal ranges. His serum lactate level was elevated to $3.0 \mathrm{mmol} / \mathrm{L}$ (normal $<2.2$ $\mathrm{mmol} / \mathrm{L}$ ) and the cerebrospinal fluid lactate level was elevated to $2.9 \mathrm{mmol} / \mathrm{L}$ (normal $<2.7 \mathrm{mmol} / \mathrm{L}$ ). WPW syndrome was revealed by electrocardiography with delta waves indicating left bundle branch block. The results of electrocardiography and a Holter electrocardiography were normal for both parents. Cardiac magnetic resonance imaging (MRI) was normal. Brain MRI showed bilateral and symmetrical signal abnormalities in the thalami and midbrain (Figure 1). The clinical phenotype combined with the elevated lactate level and cerebral lesions strongly suggested a mitochondrial encephalopathy or Leigh syndrome. DNA sequence analysis revealed that the patient had a G-to-A transition at mitochondrial DNA nt 13513 in blood specimens, resulting in an amino acid change of Asp to Asn in the mitochondrial ND5 gene by LR-PCR and next-generation sequencing (NGS, Figure 2). The mutation ratio was $86 \%$. The patient's mother either did not carry the mutations in blood specimens. There was no positive result from array comparative genomic hybridization (CGH) including the PRKAG2 gene in the family trio. Coenzyme Q10 (90 mg/d), vitamin B complex $(100 \mathrm{mg} / \mathrm{d})$, and carnitine $(300 \mathrm{mg} / \mathrm{d})$ were prescribed following the diagnosis of Leigh syndrome. The patient experienced mild improvements in ptosis. However, at the age of 23 months, he was intubated for respiratory failure caused by pneumonia and died.

\section{DISCUSSION}

The incidence of Leigh syndrome [Mendelian Inheritance in Man (MIM) 25600] is $0.025 \%$ (6), and to date, over 75 pathogenic genetic mutations have reported for Leigh syndrome (7). However, the incidences of the mutations appear to differ among specific populations.

The reported patient was diagnosed with Leigh syndrome after the onset of ptosis and developmental regression and the m.13513 G>A mutation was identified. The clinical phenotype of the G13513A mutation has significant heterogeneity. Adult onset is not uncommon, and it may appear as MELAS-Leigh syndrome or alternatively. It may be accompanied by optic atrophy, cataract, deafness and cardiac conduction dysfunction. Unlike most cases of Leigh syndrome in infants with ataxia, convulsion, peripheral nerve, and spinal cord injury, and loss of tendon reflex, the clinical phenotype in this case at presentation included only ptosis, developmental delay and Wolff-ParkinsonWhite (WPW) syndrome, which has never been described in China. Brain MRI changes showed typical symmetrical lesions in the basal ganglia and brain stem consistent with Leigh syndrome. Additionally, WPW syndrome was revealed on the patient's electrocardiogram. While the m.13513 G>A mutation first found in association with MELAS $(3,4)$, a recent report also described the m.13513 G>A mutation in cases of Leigh syndrome, especially in association with WPW syndrome (8). Leigh syndrome is commonly caused by both nuclear and mitochondrial DNA mutations of respiratory chain complex I genes (9). Kirby et al. reported that the m.13513 G>A mutation causes a complex I defect even at a low mutation level (10). Consistent with the present case, Wang et al. reported that the m.13513 G>A mutation plays an important role in Leigh syndrome and WPW syndrome, with cardiovascular involvement being common (8). Thus, screening for m.13513 


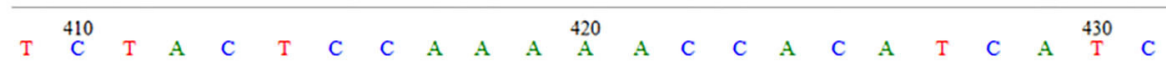

A

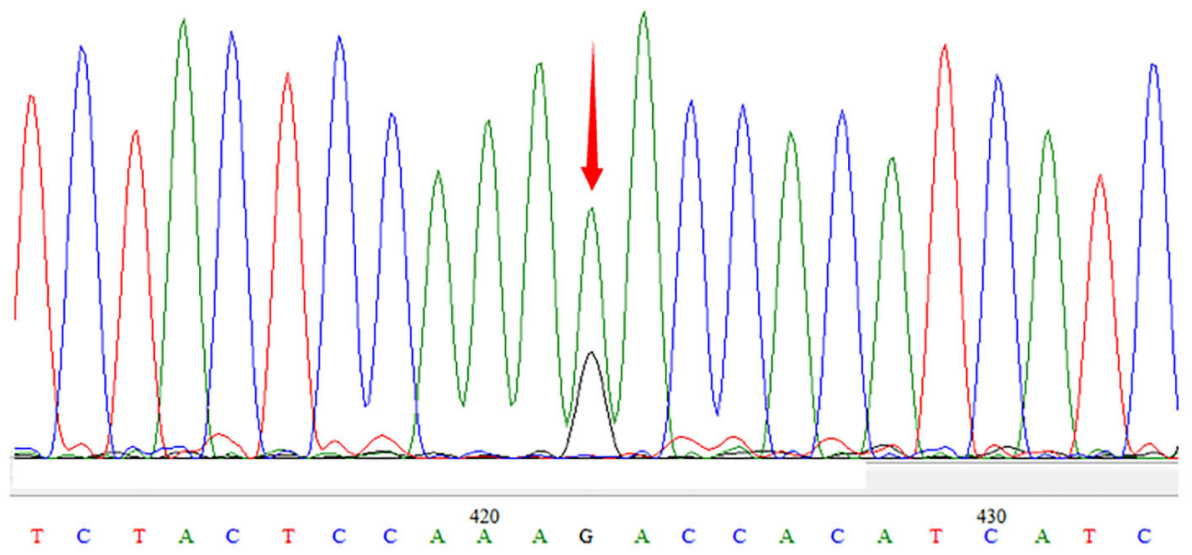

B

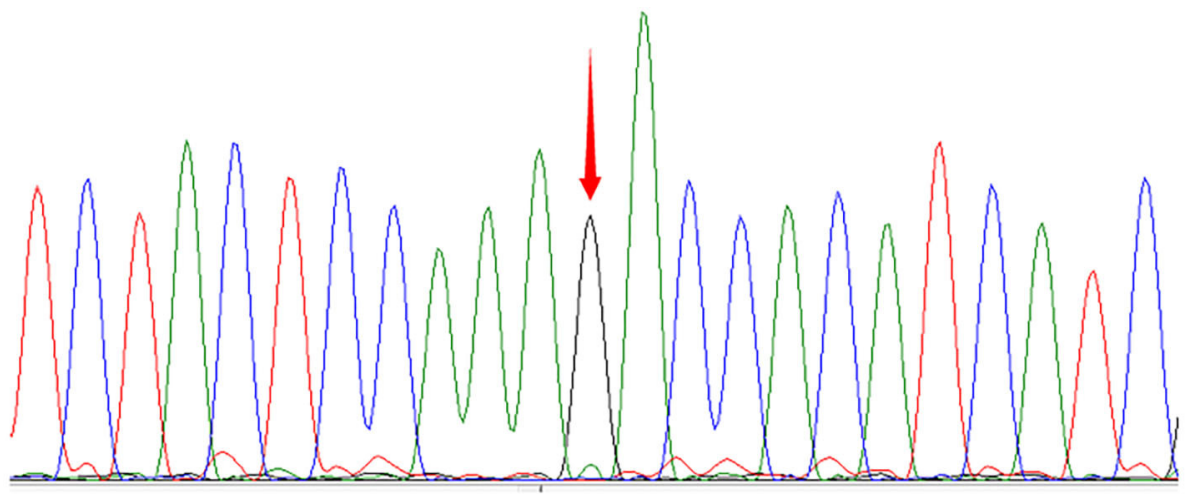

FIGURE 2 | Sequencegrams. m.13513G>A was identified in the DNA of the patient (A). The mutation was not found in DNA samples derived from his mother (B)

G $>$ A mutation should be applied in patients with Leigh syndrome and WPW syndrome.

While the m.13513 G>A mutation in Leigh syndrome in association with WPW syndrome has been described a few times in the literature, this mutation has not been reported previously in a patient in China. Yang et al. reported 65 unrelated patients who were hospitalized and diagnosed with Leigh syndrome over a 12 -year period. In their study, the clinical diagnosis of Leigh syndrome was based on clinical findings and the typical neuroradiologic findings. In $30.8 \%$ of the reported cases, the diagnosis was confirmed by genetic analysis and autopsy. SURF1 mutations were identified in 8 (12.3\%) families by DNA sequencing. The m.8344 A>G mutation was found in two cases, and the m.8993 $\mathrm{T}>\mathrm{G}$, $\mathrm{m} .8993 \mathrm{~T}>\mathrm{C}$, and $\mathrm{m} .3243 \mathrm{~A}>\mathrm{G}$ mutations were found in three cases each. Thus, in China, the SURF1 mutation has been the most commonly found mutation in Leigh syndrome (2). The present case is the first reported identification of the 
m.13513 G>A mutation in a patient with Leigh syndrome in China, and it is notable that it was found in association with WPW syndrome.

In conclusion, while Leigh syndrome has a highly variable genetic phenotype, the present case confirms the association of the m.13513 G>A mutation with both Leigh syndrome and WPW syndrome, which is the first report of this mutation in China. Therefore, the m.13513 G>A mutation should be considered in Chinese patients diagnosed with Leigh syndrome, especially those also diagnosed with WPW syndrome, highlighting the geographical, and racial variability of Leigh syndrome.

\section{DATA AVAILABILITY STATEMENT}

The raw data supporting the conclusions of this article will be made available by the authors, without undue reservation.

\section{REFERENCES}

1. Lake NJ, Bird MJ, Isohanni P, Paetau A. Leigh syndrome: neuropathology and pathogenesis. J Neuropathol Exp Neurol. (2015) 74:482-92. doi: 10.1097/nen.0000000000000195

2. Yang YL, Sun F, Zhang Y, Qian N, Yuan Y, Wang ZX, et al. Clinical and laboratory survey of 65 Chinese patients with Leigh syndrome. Chin Med J. (2006) 119:373-7.

3. Hsieh YT, Yang MT, Peng YJ, Hsu WC. Central retinal vein occlusion as the initial manifestation of LHON / MELAS overlap syndrome with mitochondrial DNA G13513A mutation-case report and literature review. Ophthalmic Genet. (2011) 32:31-8. doi: 10.3109/13816810.2010.531880

4. Folmes CD, Martinez-Fernandez A, Perales-Clemente E, Li X, McDonald A, Oglesbee D, et al. Disease-causing mitochondrial heteroplasmy segregated within induced pluripotent stem cell clones derived from a patient with MELAS. Stem Cells. (2013) 31:1298-308. doi: 10.1002/stem.1389

5. Chol M, Lebon S, Bénit P, Chretien D, de Lonlay P, Goldenberg A, et al. The mitochondrial DNA G13513A MELAS mutation in the $\mathrm{NADH}$ dehydrogenase 5 gene is a frequent cause of Leigh-like syndrome with isolated complex I deficiency. J Med Genet. (2003) 40:188-91. doi: 10.1136/jmg.40.3.188

6. Rahman S, Blok RB, Dahl HH, Danks DM, Kirby DM, Chow CW, et al. Leigh syndrome: clinical features and biochemical and DNA abnormalities. Ann Neurol. (1996) 39:343-51. doi: 10.1002/ana.4103 90311

\section{ETHICS STATEMENT}

This study was approved by the Ethics Committee of 1st hospital of Jilin University. Written informed consent to participate in this study was provided by the participants' legal guardian/next of kin.

\section{AUTHOR CONTRIBUTIONS}

J-ML was a major contributor in writing the manuscript. C-JX collected the patient data. G-LW analyzed the patient data. $\mathrm{X}-\mathrm{MW}$ revised the manuscript. All authors read and approved the final manuscript.

\section{FUNDING}

This work was funded by the National Natural Science Foundation of China (Nos. 81801284 and 81771396). The funding bodies had no role in the design of the study, the collection, analysis, or interpretation of the data, or writing the manuscript.

7. Lake NJ, Compton AG, Rahman S, Thorburn DR. Leigh syndrome: one disorder, more than 75 monogenic causes. Ann Neurol. (2016) 79:190203. doi: 10.1002/ana.24551

8. Wang SB, Weng WC, Lee NC, Hwu WL, Fan PC, Lee WT. Mutation of mitochondrial DNA G13513A presenting with Leigh syndrome, WolffParkinson-White syndrome and cardiomyopathy. Pediatr Neonatol. (2008) 49:145-9. doi: 10.1016/s1875-9572(08)60030-3

9. Shigemi R, Fukuda M, Suzuki Y, Morimoto T, Ishii E. L-arginine is effective in stroke-like episodes of MELAS associated with the G13513A mutation. Brain Dev. (2011) 33:518-20. doi: 10.1016/j.braindev.2010.07.013

10. Kirby DM, Boneh A, Chow CW, Ohtake A, Ryan MT, Thyagarajan D, et al. Low mutant load of mitochondrial DNA G13513A mutation can cause Leigh's disease. Ann Neurol. (2003) 54:473-8. doi: 10.1002/ana.10687

Conflict of Interest: The authors declare that the research was conducted in the absence of any commercial or financial relationships that could be construed as a potential conflict of interest.

Copyright (c) 2021 Liang, Xin, Wang and Wu. This is an open-access article distributed under the terms of the Creative Commons Attribution License (CC BY). The use, distribution or reproduction in other forums is permitted, provided the original author(s) and the copyright owner(s) are credited and that the original publication in this journal is cited, in accordance with accepted academic practice. No use, distribution or reproduction is permitted which does not comply with these terms. 